Litvinchuk et al. observed high levels of $\mathrm{C} 3$ and the $\mathrm{C} 3 \mathrm{a}$ receptor (C3aR) - which were mainly co-localized with astrocytes and microglia, respectively - in the PS19 mice. Similarly, C3 and C3aR1 mRNA levels were increased in the parahippocampal gyrus of individuals with $\mathrm{AD}$, and correlated with cognitive decline. PS19 mice also showed increased astrogliosis and microgliosis, suggesting that PS19 mice exhibit neuroinflammation.

Litvinchuk et al. next found that PS19 mice lacking C3aR (PS19;

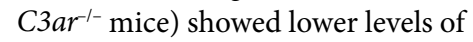
astrocytic C3, a microglial lysosome marker and pro-inflammatory cytokines than did PS19 mice. The deletion of C3ar from PS19 mice also prevented the development of fear-learning deficits and reductions in the number of hippocampal neurons and synapses. RNAsequencing analysis identified 1,726 differentially expressed genes (DEGs) in PS19 mice versus controls; strikingly, the expression of 526 of these DEGs was normalized by C3ar deletion. Notably, 301 genes

when mice were treated with a secretin-neutralizing antibody. Similarly, in a sample of 17 human volunteers, secretin levels rose rapidly after eating, as did oxygen consumption rates (indicating thermogenesis).

In mice, peaks in BAT temperature occurred just before the end of feeding, suggesting that BAT thermogenesis may suppress appetite. Indeed, an injection of exogenous secretin immediately before feeding reduced food intake, whereas injection of the secretin antibody stimulated food intake.

These results suggest that BAT thermogenesis activates central satiation pathways. Indeed, the authors showed that secretin increased the levels of the anorexigenic hormone precursor pro-opiomelanocortin (POMC) and decreased expression of the orexigenic hormone agouti-related protein in the hypothalamus of mice.

Secretin also increased the expression of the temperature-sensitive ion channel transient receptor potential vanilloid 1 (TRPV1) in hypothalamic POMC-expressing neurons, suggesting a mechanism for the detection of whose expression was correlated with $C 3 a R$ expression in human brain data sets were downregulated by C3ar deletion in PS19 mice. One of these genes encodes signal transducer and activator of transcription 3 (STAT3), a proinflammatory transcription factor. Litvinchuk et al. confirmed in cultured microglia that $\mathrm{C} 3-\mathrm{C} 3 \mathrm{aR}$ binding regulates STAT3 activation. Treating PS19 mice with a STAT3 inhibitor reduced astrogliosis, microgliosis and the levels of inflammatory cytokines; thus, STAT3 may contribute to neuroinflammation in PS19 mice.

Together, these studies indicate that tau pathology drives the phagocytosis of synapses and neuroinflammation via the complement system.

Natasha Bray

ORIGINAL ARTICLES Litvinchuk, A. et al. Complement $\mathrm{C} 3 \mathrm{a}$ R inactivation attenuates tau pathology and reverses an immune network deregulated in tauopathy models and Alzheimer's disease. Neuron https://doi.org/10.1016/j.neuron. 2018.10.031 (2018)| Dejanovic, B. et al. Changes in the synaptic proteome in tauopathy and rescue of tau-induced synapse loss by $\mathrm{C} 1 \mathrm{q}$ antibodies. Neuron https://doi.org/10.1016/j.neuron.2018. 10.014 (2018)

a rise in local temperature by these neurons.

This study reveals a key role for BAT in conveying information from the gut to the brain to control appetite and adds to our understanding of the complex mechanisms that regulate energy balance. The contribution of BAT thermogenesis to appetite suppression, as well as its known effect on energy expenditure, suggests it is a possible target for obesity therapeutics. However, the authors found that chronic treatment of mice with secretin did not alter overall body weight, suggesting that compensatory mechanisms act to limit its effects. Thus, a more nuanced approach may be required to exploit this discovery for therapeutic benefit.

Katherine Whalley

ORIGINAL ARTICLE Li, Y. et al. Secretinactivated brown fat mediates prandial thermogenesis to induce satiation. Cell. https://doi.org/10.1016/j.cell.2018.10.016 (2018) FURTHER READING Kim, K.-S. et al. Signalling from the periphery to the brain that regulates energy homeostasis. Nat. Rev. Neurosci. 19, 185-196 (2018)
IN BRIEF

\section{$\Rightarrow$ TECHNIQUES}

\section{Gone FISHing}

The molecular-cellular architecture of the hypothalamic preoptic area is not well understood. Here, more than 1 million cells from the preoptic region of mouse hypothalamus were analysed using single-cell RNA sequencing (scRNAseq) and multiplexed error-robust fluorescence in situ hybridization (MERFISH). scRNAseq provided a detailed analysis of gene expression in individual cells, and MERFISH enabled gene expression profiling of, and imaging of activity markers in, live cells, thus providing a spatial context to the expression patterns at high resolution. Applying these techniques after various behaviours known to involve the preoptic area revealed differences in FOS expression patterns in $\sim 70$ specific neuronal populations, deepening our understanding of this complex brain area.

ORIGINAL ARTICLE Moffitt, J. R. et al. Molecular, spatial and functional single-cell profiling of the hypothalamic preoptic region. Science 362, eaau5324 (2018)

\section{$\Rightarrow$ TECHNIQUES}

\section{Patchwork subiculum}

The primary output pathway of the hippocampus is provided by the pyramidal cells of the subiculum. Here, analysis of mouse subiculum using single-cell RNA sequencing revealed widespread differences in gene expression that could be mapped onto eight distinct subpopulations of pyramidal cell. Further analysis with in situ hybridization showed that these subclasses were spatially partitioned into specific domains that corresponded to specific downstream projection targets. This more detailed picture of the complex landscape of the subiculum is likely to aid future study of this area.

ORIGINAL ARTICLE Cembrowski, M. S. et al. The subiculum is a patchwork of discrete subregions. elife 7, e37701 (2018)

\section{$\Rightarrow$ TECHNIQUES}

\section{Closing the loop}

Here, an 'all optical' combination of techniques was used to investigate how spatiotemporal brain activity patterns drive behaviour. Optical readout from a genetically encoded calcium indicator obtained in real time from mouse barrel cortex was used to infer population activity; these data were used to reconfigure the rate and pattern of barrel-cortex photostimulation rapidly enough for ongoing patterns of sensory-evoked activity in individual neurons to be rapidly enhanced in the awake mouse in real time.

ORIGINAL ARTICLE Zhang, Z. et al. Closed loop all-optical interrogation of neural circuits in vivo. Nat. Methods https://doi.org/10.1038/s41592-018-0183-z (2018)

\section{$\Rightarrow$ LEARNING AND MEMORY}

\section{To sleep, to remember}

Some forms of memory, such as novel-object recognition (NOR), can be acquired and retrieved without involving the hippocampus. Rats were exposed to a novel object (the encoding period) followed by a 2-hour period of either wakefulness or sleep. Three weeks after encoding, NOR memory in rats was retained in the sleep condition but not in the wake condition. The effect of sleep on NOR retrieval was abolished by hippocampal inactivation by muscimol delivered during post-encoding sleep but not before encoding or before retrieval. These findings suggest that sleep-mediated consolidation of NOR memory involves the hippocampus. ORIGINAL ARTICLE Sawangjit, A. et al. The hippocampus is crucial for forming non-hippocampal long-term memory during sleep. Nature https://doi.org/10.1038/ s41586-018-0716-8 (2018) 